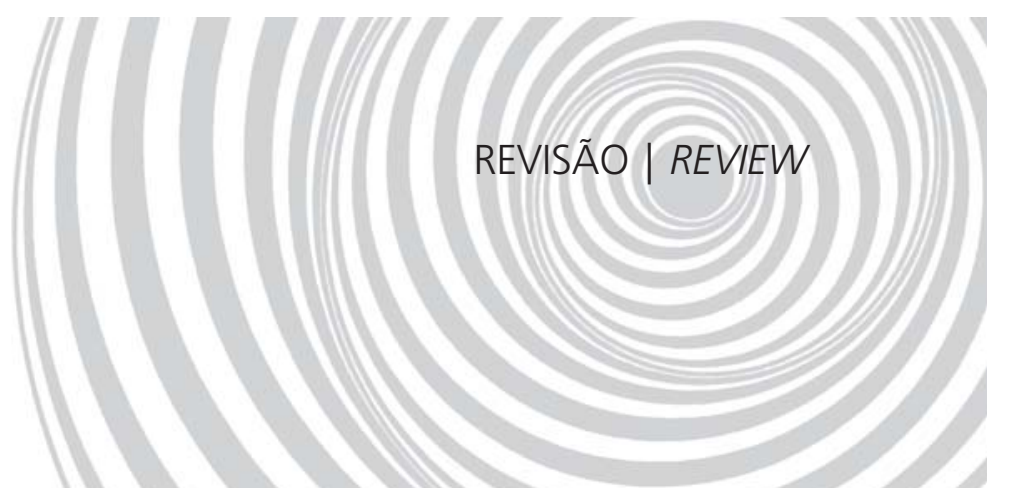

\title{
Abordagem metabólica e nutricional da lipodistrofia em uso da terapia anti-retroviral
}

\author{
Metabolic and nutritional approach of lipodystrophy \\ in the use of antiretroviral therapy
}

Claudia Daniele Tavares DUTRA

Rosana Maria Feio LIBONATI'

A terapia anti-retroviral altamente ativa, usada contra o Vírus da Imunodeficiência Humana, vem possibilitando a melhora do quadro clínico-laboratorial de portadores da Síndrome da Imunodeficiência Adquirida. Contudo, alterações metabólicas e complicações morfológicas, associadas ao uso da terapia, vêm sendo investigadas. A utilização prolongada desta terapia tem um impacto importante sobre o estado nutricional dos pacientes. Antes da sua utilização, a perda de peso e a desnutrição, conseqüências das infecções oportunistas, eram os maiores problemas nutricionais. Atualmente, o foco principal das discussões têm sido as complicações metabólicas e morfológicas, dentre elas a lipodistrofia, com a dislipidemia, a resistência à insulina, a osteopenia, e a distribuição alterada da gordura corporal, aumentando assim os riscos de doenças cardiovasculares. A nutrição desempenha um papel fundamental no suporte da saúde desses pacientes, integrando as equipes multiprofissionais, promovendo a melhora da adesão à terapia anti-retroviral e do prognóstico da doença. No entanto, para que se tenha mais conhecimento sobre a terapia, as proporções de seus efeitos adversos, e o perfil nutricional desses pacientes, a curto e a longo prazos, é de suma importância que se estude mais sobre este assunto, a fim de permitir perspectivas de um regime terapêutico mais seguro dentro de seus alcances metodológicos, proporcionando uma melhor qualidade de vida aos pacientes.

Termos de indexação: Lipodistrofia. Nutrição. Terapia anti-retroviral de alta atividade.

A B S T R A C T

The highly active antiretroviral therapy used against Human Immunodeficiency Virus provides an improvement in laboratory and clinical findings of patients with Acquired Immunodeficiency Syndrome. However, metabolic and morphologic disturbances associated with the therapy are being investigated. The drawn out use of these therapy has an important impact on the nutritional status of the patients. Before the use of this therapy, weight loss and malnutrition caused by opportunistic infections were the biggest nutritional problems.

${ }^{1}$ Universidade Federal do Pará, Centro de Ciências da Saúde, Núcleo de Medicina Tropical. Av. Generalíssimo Deodoro, 92, Umarizal, 66055-240, Belém, PA, Brasil. Correspondência para/Correspondence to: R.M.F. LIBONATI. E-mail: $<$ fe_any@terra.com.br> 
440 | C.D.T. DUTRA \& R.M.F. LIBONATI

Nowadays the main discussion points are the resulting metabolic and morphologic complications, among them lipodystrophy with dyslipidemia, insulin resistance, osteopenia and altered distribution of body fat, thus increasing the risk of cardiovascular diseases. Nutrition plays an essential role in supporting the health of these patients, integrating the multidisciplinary teams, improving antiretroviral therapy adherence rates and disease prognosis. However, in order to better understand the therapy, the side effect rates and the nutritional profile of these patients on the short and long run, it is vital to study this subject more deeply to obtain perspectives of a safer therapeutic regimen within its methodological scope, improving the quality of life of these patients.

Indexing terms: Lipodystrophy. Nutrition. Antiretroviral therapy, highly active.

\section{N T R O D U ÇÃ O}

A epidemia da síndrome da imunodeficiência adquirida (AIDS) continua crescendo, apesar do declínio das taxas de infecção. Segundo as Nações Unidas, em sua atualização de 2006, o número global de pessoas vivendo com HIV (Vírus da Imunodeficiência Humana) vem aumentando, em todas as regiões do mundo, com o número estimado de novos casos em adultos e crianças de 4,3 milhões. O número de portadores de HIV aproxima-se de 39,5 milhões de pessoas, sendo 2,6 milhões a mais que em 2004, e que a mortalidade foi de 2,9 milhões de pessoas naquele ano' ${ }^{1}$.

No Brasil, estima-se que tenham ocorrido 433 mil casos de AIDS, evidenciados desde a primeira identificação, em 1980, até junho de 2006. A taxa de incidência aumentou, atingindo em 1998, 19 casos de AIDS por 100 mil habitantes. Em 2004, foram notificadas 593 mil pessoas, entre 15 a 49 anos de idade, que vivem com HIV e AIDS, sendo 208 mil mulheres e 385 mil homens. A mortalidade até dezembro de 2005 atingiu o número de 183 mil óbitos por AIDS, estabilizando em cerca de 11 mil óbitos anuais desde 19982,3.

Após o progresso da política de acesso universal ao tratamento anti-retroviral (TARV), que levou à introdução da terapia anti-retroviral altamente ativa (HAART), com a combinação de drogas com diferentes formas de ação, observou-se uma importante redução na mortalidade dos pacientes infectados pelo HIV3,4.

Com a utilização do esquema HAART, a replicação do HIV é inibida, com diminuição da presença do RNA do HIV no plasma para níveis indetectáveis, assim prolongando a sobrevida do paciente ${ }^{5}$. No entanto, o esquema é complexo e apresenta muitas alterações metabólicas ${ }^{4}$.

A introdução da HAART mudou o estado nutricional dos pacientes com HIV. Antes do tratamento anti-retroviral, principalmente com uso de Inibidores de Protease (IP), os déficits de vitaminas e minerais e a má nutrição energético-protéica estavam associados como os maiores problemas nutricionais da época, sendo a má nutrição energético-protéica responsável por $80 \%$ da mortalidade dos pacientes com AIDS ${ }^{6}$.

Atualmente, com os avanços no tratamento anti-retroviral, as pessoas portadoras de HIV comumente apresentam ao longo da infecção uma variedade de problemas nutricionais como perda de peso, redistribuição de gordura e obesidade. A incidência da desnutrição tende a aumentar, devido à sobrevivência prolongada, sendo essencial monitorar o peso dos pacientes assintomáticos, sintomáticos ou com modificações no peso ${ }^{7}$.

\section{Tratamento}

A HAART continua sendo indicada para todos os pacientes infectados pelo HIV, sintomáticos ou assintomáticos, que apresentam contagem de linfócitos T CD4+ abaixo de 200/ $\mathrm{mm}^{3}$. No caso de paciente assintomático que apresentar contagem de linfócitos T CD4+ entre 200 e $350 / \mathrm{mm}^{3}$, o início da HAART deve ser considerado de acordo com a evolução do quadro clínico do paciente como os parâmetros imunológicos, virológicos, a motivação, a adesão e as co-morbidades $^{8}$. 
Embora o uso potente da HAART tenha diminuído a mortalidade e a progressão da doença, em pacientes infectados com HIV, a morbidade secundária a esta terapia tem crescido a longo prazo 9 .

O principal objetivo da HAART é retardar a progressão da imunodeficiência e/ou restaurar, tanto quanto possível, a imunidade, aumentando o tempo de vida da pessoa infectada ${ }^{5}$.

As classes de drogas liberadas para o tratamento anti-HIV são: inibidores de transcriptase reversa análogos de nucleosídeo (ITRN); inibidor de transcriptase reversa não nucleosídeo (ITRNN), que são drogas que inibem a replicação do vírus HIV bloqueando a enzima transcriptase reversa, que age copiando o RNA (ácido ribonucléico) viral em DNA (ácido desoxirribonucléico) e as drogas inibidoras da protease viral (IP), que são drogas que agem no último estágio na formação do HIV, impedindo a ação da enzima protease ${ }^{10}$.

Entre as diversas complicações da HAART destacam-se: lipodistrofia, dislipidemia, resistência à insulina, osteopenia, alterações glicêmicas e cardíacas como os efeitos colaterais mais preocupantes. Além do que, a alteração na composição da gordura corporal prejudica a auto-imagem e pode interferir na adesão à terapia, levando à falha terapêutica ${ }^{11,12}$.

\section{Lipodistrofia}

As complicações metabólicas, incluindo dislipidemia, resistência à insulina e distribuição da gordura corporal (perda do tecido adiposo subcutâneo e um relativo aumento da gordura visceral) são comuns em adultos infectados pelo HIV em uso da HAART, podendo ser conjuntas ou independentes, sugerindo um complexo de causas multifatoriais, aumentando, assim, o risco de doenças cardiovasculares ${ }^{13,14}$.

O tipo, a duração e o uso ou não da HAART estão fortemente associados com a gravidade da lipoatrofia. A terapia combinada, baseada no uso de dois análogos nucleosídeos inibidores da transcriptase reversa e de um inibidor da protease, está fortemente associada com a lipoatrofia grave $^{13}$.

Para o diagnóstico da lipodistrofia podem ser utilizadas medidas antropométricas, absormetria com duplo feixe de Raios $X$, tomografia computadorizada e ressonância nuclear magnética ${ }^{15}$. Para avaliação da dislipidemia empregamse as dosagens dos níveis de colesterol total, de suas frações e de triglicerídeos. Para avaliação da resistência à insulina e o diagnóstico de diabetes são úteis a realização de glicemia de jejum, de curva glicêmica e a dosagem da insulinemia basal ${ }^{11}$.

Atualmente não existe tratamento padrão para nenhum componente da síndrome lipodistrófica e a decisão do tratamento vai depender de algumas variáveis como: presença de sintomas, quadro clínico, tipo de anti-retrovirais utilizados, tempo de uso da medicação e presença de um ou mais fatores de risco cardiovascular ${ }^{4}$.

Estudos com o hormônio do crescimento têm revelado redução na obesidade de tronco e na "giba de búfalo" após 3 a 6 meses de tratamento, mas nenhuma reversão da perda de gordura periférica. Exercícios físicos podem reduzir a massa gordurosa total, inclusive a localizada no tronco $^{16}$.

\section{Alterações dos lipídeos}

A dislipidemia em níveis associados com o aumento do risco de doenças cardiovasculares ocorre em, aproximadamente, $70 \%$ dos pacientes infectados pelo HIV-1 recebendo a HAART ${ }^{17}$.

Tsiodras et al. ${ }^{18}$, em sua pesquisa, observaram que a hipercolesterolemia e/ou hipertrigliceridemia está associada ao uso de IP. Após cinco anos de seguimento de pacientes HIV positivos, em terapia com IP verificou-se uma incidência cumulativa de $20 \%$ de casos de hipercolesterolemia e hipertrigliceridemia. Outro estudo ${ }^{19}$ demonstrou que um grupo de pacientes que nunca recebeu $\mathbf{I}$, e que estava em tratamento com 
inibidores de transcriptase reversa análogos de nucleosídeo, apresentou níveis elevados de triglicerídeos, sugerindo a existência de outros fatores $^{11}$.

É importante a investigação de história familiar para dislipidemia e/ou diabetes, bem como dos hábitos de vida do paciente, como uso de álcool ou medicamentos como estrógeno. Dessa forma, a mudança no estilo de vida torna-se indispensável no tratamento da dislipidemia. Nos casos de associação entre as drogas anti-retrovirais e antilipemiantes é fundamental uma rigorosa monitorização da função renal, das enzimas hepáticas e da creatinofosfoquinase pela potencialização dos efeitos nefrotóxicos, hepatotóxicos e miotóxicos destas medicações ${ }^{11,13}$.

\section{Carboidratos}

O primeiro registro de casos de hiperglicemia em pacientes HIV foi feito em 1997, com a ocorrência de 83 pacientes em uso de terapia antiretroviral com IP. Um estudo de corte transversal verificou que a lipodistrofia, a hiperlipidemia e a resistência insulínica eram complicações comuns dos IP nos pacientes HIV20.

Pesquisa sugere que os pacientes HIV positivos com normoglicemia apresentam diminuição no metabolismo da glicose e dos lipídios em múltiplas vias envolvendo fígado, tecido muscular e alterações funcionais das células $\beta$ pancreáticas $^{21}$.

A prevalência de diabetes é quatro vezes mais comum em homens em terapia anti-retroviral, comparados com não infectados. A incidência de pré-diabetes e diabetes é de duas e três vezes, respectivamente ${ }^{22}$.

O tratamento de indivíduos não infectados pelo HIV com indinavir (IP) promoveu instalação rápida de resistência insulínica, sem mudanças na composição corporal. No entanto, é importante salientar que a resistência insulínica pode estar associada à própria infecção pelo HIV, provavelmente, pela ação direta do vírus na função das células $\beta$ pancreáticas, como nos mecanismos de secreção insulínica"11.

O diabetes parece também contribuir para seqüelas neurológicas das infecções por HIV. Um estudo de coorte examinou os fatores associados com várias funções cognitivas em adultos infectados por HIV e encontrou que o diabetes foi mais comum entre pessoas idosas, sendo este associado à piora global das funções cognitiva e psicomotora, atribuído ao uso da HAART, a níveis de colesterol elevados e ao fumo 22,23 .

\section{Osteopenia e osteoporose}

A prevalência da diminuição da densidade mineral óssea em adultos infectados pelo HIV tem sido de $22 \%-50 \%$ de adultos com osteopenia e $3 \%-21 \%$ com osteoporose ${ }^{24}$.

A osteopenia no paciente HIV tem sido relacionada com a terapia anti-retroviral, ressaltando a perda da densidade mineral óssea, associada ao baixo peso antes do início da terapia e à acidemia láctica devido aos inibidores de transcriptase reversa análogos de nucleosídeos ${ }^{20}$.

Nos pacientes com osteoporose devem ser investigadas outras possíveis causas associadas a esse processo, como tirotoxicose, hiperparatiroidismo, hipogonadismo, etilismo, má-absorção, inatividade física, perda de peso recente, utilização de medicamentos como corticosteróides, fenobarbital, pentamidina ou cetoconazol. Além de que os pacientes devem ingerir uma quantidade suficiente de cálcio e vitamina D em forma de alimentos ou suplementação. Em determinados casos pode-se prescrever também difosfonatos ${ }^{25}$.

\section{A LTERAÇÕES NUTRICIONAIS ASSOCIADOS À HAART}

\section{Avaliação nutricional}

A avaliação nutricional observa as deficiências isoladas ou globais de nutrientes e classifica os indivíduos quanto ao seu estado nutricional, 
agindo como instrumento de grande valia para a terapêutica clínica ou dietética, a fim de tentar corrigir o déficit diagnosticado ${ }^{12}$.

A avaliação do estado nutricional requer a análise de parâmetros antropométricos, clínicos e bioquímicos, resultando na classificação e na avaliação dos pacientes em função de sua situação nutricional ${ }^{26}$.

Como instrumentos de avaliações antropométricas destacam-se: o índice de massa corporal (IMC), a circunferência da cintura como indicador de obesidade abdominal, as medidas de pregas cutâneas e a análise por bioimpedância7, 27 .

\section{Alteração de peso}

Durante as perdas ponderais, o valor energético total gasto fica reduzido, devido à ingestão inadequada, aos problemas absortivos e ao aumento das necessidades energéticas ${ }^{28}$.

Estudo transversal com pacientes de HIV em tratamento com HAART, no município de São Paulo, concluiu que a obesidade destacou-se como o desvio do estado nutricional mais importante, superando a desnutrição, nessa população ${ }^{29}$.

No exercício da HAART, é importante que os pacientes com HIV gerenciem os componentes do balanço energético através do acompanhamento nutricional, uma vez que estes estão facilmente vulneráveis às alterações do peso ${ }^{7}$.

\section{Objetivos da dietoterapia}

Os objetivos da dietoterapia em relação aos pacientes com AIDS são: preservar a massa magra; evitar a desnutrição; recuperar o estado nutricional; fornecer quantidades adequadas de nutrientes; reduzir as complicações, e os sintomas de infecções oportunistas, os efeitos colaterais de drogas que interfiram na ingestão e absorção de nutrientes; assim como melhorar a qualidade de vida dos pacientes ${ }^{30,31}$.

\section{Recomendações nutricionais}

A Organização Mundial de Saúde preconiza que as intervenções nutricionais façam parte de todos os programas de controle e tratamento da AIDS, pois a dieta e a nutrição podem melhorar a adesão e a efetividade da terapia anti-retrovira ${ }^{32}$.

Como a síndrome lipodistrófica é muito recente, ainda não há manejo clínico nutricional padronizado, mas sabe-se que as recomendações como alimentação saudável e aderência do paciente ao tratamento são muito importantes ${ }^{33}$.

A educação nutricional é uma etapa essencial no suporte nutricional, que busca prevenir o estado de má nutrição, de acordo com os critérios de segurança alimentar, proporcionando a energia e os nutrientes necessários ${ }^{34}$.

O consumo de uma variedade de alimentos é fundamental para uma alimentação balanceada. Segundo a pirâmide de alimentos recomendamse: 6-11 porções por dia de grãos, (como pão, cereais, arroz e massas); 3-5 porções de legumes; 2-4 porções de frutas; 2-3 porções de produtos lácteos (como leite, iogurte e queijo); 2-3 porções de proteína (como carne, aves, peixes, ovos e leguminosas) e pequenas quantidades de gordura, óleo e açúcar ${ }^{28}$.

A estimativa do gasto metabólico basal pode ser realizada por meio da fórmula de Harris Benedict ou pelos cálculos das necessidades energéticas simplificadas a partir de $35-40 \mathrm{kcal} / \mathrm{kg} / \mathrm{dia}^{35}$. Pode-se, ainda, calcular a demanda energética levando em consideração os sintomas do paciente, por exemplo: os assintomáticos devem aumentar $10 \%$ de energia sobre a sua ingestão, enquanto que, os sintomáticos, $20 \%$ a $30 \%$, ambos calculados, conforme a recomendação energética de pacientes saudáveis não infectados por HIV, para mesma idade, sexo e nível de atividade física ${ }^{36}$.

A ingestão de carboidratos, principalmente os complexos, deve representar, pelo menos, 50\% da ingestão total energético diário ${ }^{28}$.

Os pacientes com diabetes e/ou resistência à insulina devem regular sua glicemia com dietas 
específicas, diminuindo carboidratos simples (doces) e aumentando carboidratos complexos (pães, massa, batata, mandioca) de forma equilibrada ${ }^{33}$.

A recomendação para ingestão de proteínas é de $15 \%$ a $20 \%$ do total de energia da $\operatorname{dieta}^{28}$. No entanto, muitos especialistas utilizam o critério de cálculos a partir de $1 \mathrm{~g} / \mathrm{kg}$ de peso por dia, sendo que em situação de estresse catabólico pode ser necessário aumentar o aporte de 1,5 a 2,0 $/ \mathrm{kg} / \mathrm{dia}^{35}$. Em casos de problemas renais ou hepáticos deve-se realizar um monitoramento rigoroso do consumo de proteínas ${ }^{33}$.

As recomendações dietéticas em caso de hipercolesterolemia preconizam colesterol menor que $200 \mathrm{mg} / \mathrm{dia}$, gordura saturada menor que $7 \%$ do total de calorias/dia e a redução da gordura total para até $30 \%$. Dietas hiperlipídicas aumentam as chances de desenvolvimento de doenças cardiovasculares e de alguns tipos de cânceres ${ }^{37}$.

Quando os resultados dos exames de colesterol e triglicerídeos estão elevados, há indicação para modificações dietéticas, como redução e substituição das gorduras saturadas por gorduras monoinsaturadas e poliinsaturadas e ácidos graxos ômega- $3^{38}$. Dependendo do caso, se necessário, faz-se uso de hipolipemiantes para melhorar o quadro de dislipidemia ${ }^{33}$.

A suplementação de micronutrientes pode estar recomendada em situações especiais de máabsorção de selênio, zinco, vitaminas A e do complexo $B$, cujo déficit está associado à piora progressiva da resposta imunológica. No entanto, intervenções específicas têm reportado resultados muito variáveis, sendo desejáveis aportes multivitamínicos e minerais não suprafisiológicos que podem ser obtidos por uma dieta equilibrada ${ }^{39}$. A superdosagem de micronutrientes pode ser prejudicial à saúde, doses máximas de consumo habitual devem ser observadas, a fim de prevenir a ocorrência de efeitos adversos. As vitaminas $A$, $E, C$ e $B_{6}$, assim como os minerais, selênio, zinco e cálcio podem ser tóxicos em altas doses, por isso deve-se obedecer ao limite máximo tolerável para ingestão desses micronutrientes ${ }^{37}$.
Os pacientes podem apresentar fatores de riscos múltiplos para perda da densidade mineral óssea, devendo consumir alimentos ricos em proteína e vitamina $D$, aumentar a massa magra, evitar tabagismo, álcool, cafeína, balancear o consumo dos alimentos e das bebidas elevadas em ácido fosfórico, preferindo os ricos em cálcio em substituição às bebidas carbonatadas ${ }^{40,41}$. Dependendo do caso, se necessário, realizar tratamento medicamentoso para a osteoporose ${ }^{33}$.

A prática de exercícios físicos tem demonstrado seus benefícios, produzindo o aumento da força muscular com um ganho progressivo de peso e massa celular corporal, assim como melhora do perfil lipídico ${ }^{42}$.

Dentre algumas recomendações dietéticas, destacam-se ${ }^{38}$ : aumentar o consumo de alimentos ricos em fibras (como grãos, frutas e legumes); reduzir o consumo dos carboidratos refinados (como pães e arroz brancos, tortas e biscoitos) e aumentar os carboidratos com alto teor de fibras (como farelo de trigo, aveia, cereais e pães integrais); diminuir e substituir o consumo de gorduras saturadas (gorduras animais) e gorduras trans (presentes no preparo de bolos, biscoitos e lanches rápidos), pelos benefícios das gorduras monoinsaturadas (óleo de oliva, abacate, amêndoas, nozes) e gorduras poliinsaturadas (nozes e sementes, óleo de soja e os alimentos ricos em ômega-3); aumentar a ingestão de peixes, que contenham ômega-3 e ácidos graxos (como salmão, atum, sardinha); praticar exercícios físicos regularmente; parar de fumar.

\section{CONSIDERAÇÕ ES FINAIS}

A eficácia da utilização da HAART é evidente no combate ao HIV, logo cabe ressaltar a importância da manutenção do estado nutricional do paciente, que terá como desafio empregar a dietoterapia para otimizar a absorção de nutrientes e favorecer a eliminação e a tolerância à medicação' ${ }^{12}$.

A intervenção nutricional desempenha um papel fundamental no suporte da saúde desses 
pacientes, integrando as equipes multiprofissionais, promovendo a integralização da saúde, a adesão à terapia anti-retroviral e melhorando o prognóstico da doença ${ }^{12}$.

Para que se tenha mais conhecimento sobre a terapia anti-retroviral, as proporções de seus efeitos adversos e o perfil nutricional nesses pacientes, a curto e a longo prazo, é de suma importância que se pesquise mais sobre este assunto, a fim de permitir perspectivas de um regime terapêutico mais seguro dentro de seus alcances metodológicos, proporcionando uma melhor qualidade de vida aos pacientes.

\section{COLABORADORES}

C.D.T. DUTRA participou da elaboração do artigo e da revisão dos dados das alterações nutricionais associados à HAART. R.M.F. LIBONATI revisou as alterações metabólicas relacionadas ao uso da HAART.

\section{REFERÊ N CIAS}

1. United Nations Programme on HIV/AIDS. AIDS epidemic Update: special report on HIV/AIDS. Geneva: WHO; 2006.

2. Brasil. Ministério da Saúde. DST/AIDS: AIDS em números [acesso 2007 fev 22]. Disponível em: <http://www.aids.gov.br/data/Pages/LUMIS13F 4BF21PTBRIE.htm>.

3. Brasil. Ministério da Saúde. DST/AIDS: Epidemiologia [acesso 2007 fev 22]. Disponível em: <http://www.aids.gov.br/data/Pages/LUMISD 3352823PTBRIE.htm>.

4. Oliveira S, Lins D. Lipodistrofia relacionada à síndrome de imunodeficiência adquirida (AIDS). In: Bandeira F, Macedo G, Caldas G, Griz L, Faria MS. Endocrinologia e diabetes. Rio de Janeiro: Medsi; 2003. p.1018-22.

5. Rang HP, Dale MM, Ritter JM. Farmacologia. 4a. ed. Rio de Janeiro: Guanabara Koogan; 2001. p.595-604.

6. Scevola D, Matteo DA, Uberti F, Minoia G, Poletti F, Faga A. Reversal of cachexia in patients treated with potent antiretroviral. AIDS Read. 2000; 10(6): 365-75.

7. Shevitz AH, Knox TA. Nutrition in the era of highly active antiretroviral therapy. Clin Infect Dis. 2001; 32(12):1769-75.
8. Brasil. Ministério da Saúde. Recomendações para terapia anti-retroviral em adultos e adolescentes infectados pelo HIV. Brasília; 2003.

9. Fernandes APM, Sanches SER, Porfírio E, Machado AA, Donadi EA. Lipodistrofia em portadores do HIV. J Bras AIDS. 2005; 6(3):93-140.

10. World Health Organization. Terapia anti-retroviral para o tratamento da infecção pelo HIV [acesso 2007 fev 22]. Disponível em: <http://whqlibdoc. who.int/hq/2004/WHO_HTM_TB_2004.329_ por_chap11.pdf>.

11. Valente AMM, Reis AF, Machado DM, Succi RCM, Chacra AR. HIV lipodystrophy syndrome. Arq Bras Endocrinol Metab. 2005; 49(6):871-81.

12. American Dietetic Association. Position of the american dietetic association and dietitians of Canada: nutrition intervention in the care of persons with human immunodeficiency virus infection. J Am Diet Assoc. 2004; 104(9):1425-41.

13. Grinspoon S, Carr A. Cardiovascular risk and body-fat abnormalities in HIV-infected adults. N Engl J Med. 2005; 352(1):48-62.

14. Salyer J, Lyon DE, Settle J, Elswick RK, Rackley D. Coronary heart disease risks and lifestyle behaviors in persons with HIV infection. J Assoc Nurses AIDS Care. 2006; 17(3):3-17.

15. Currier J, Carpenter C, Daar E, Kotler D, Wanke C. Identifying and managing morphologic complications of HIV and HAART. AIDS Read. 2002; 12(3):114-25.

16. Castellar E, Vilar L. Distúrbios endócrinos e metabólicos na AIDS. In: Vilar L, Castellar E, Moura E, Leal E, Machado AC, Teixeira L, et al. Endocrinologia clínica. 2a. ed. Rio de Janeiro: Medsi; 2001. p.871-82.

17. Montessori V, Press N, Harris M, Akagi L, Montaner JSG. Adverse effect of antiretroviral therapy for HIV infection. Can Med Assoc J. 2004; 170(2):229-38.

18. Tsiodras S, Mantzoros C, Hammer S, Samore M. Effects of protease inhibitors on hyperglycemia, hiperlipidemia ande lipodystrophy- a 5 years cohort study. Arch Int Med. 2000; 160(13):2050-6.

19. Madge S, Kinloch-de-Loes S, Mercey D, Johnson MA, Weller IVD. Lipodystrophy in patients naive to HIV protease inhibitors [correspondence]. AIDS. 1999; 13(6):735-7.

20. Carr A, Samaras K, Burton S, Law M, Freund J, Chisholm D, et al. A syndrome of peripheral lipodystrophy, hiperlipidaemia and insulin resistance in patients receiving HIV protease inhibitors. AIDS. 1998; 12(7):F51-8.

21. Haugaard SB, Andersen O, Storgaard H, Dela F, Holst JJ, Iversen J, et al. Insulin secretion in 
lipodystrophic hiv patients is associated with high levels of nonglucose secretagogues and insulin resistance of beta-cells. Am J Physiol Endocrinol Metab. 2004; 287(4):E677-E85.

22. Currier JS, Havlir DV. Complication of HIV disease and antiretroviral therapy. Top HIV Med. 2004; 12(1):31-9.

23. Sweet DE. Metabolic complication of antiretroviral therapy. Top HIV Med. 2005; 13(2):70-4.

24. Lima GAB, Verdeal JCR, Farias MLF. Osteonecrosis in patients with acquired immunodeficiency syndrome (AIDS): Report of Two Cases and Review of Literature. Arq Bras Endocrinol Metab. 2005; 49(6):996-9.

25. Roca B. Trastornos metabólicos relacionados con el VIH y el tratamiento antirretroviral. An Med Interna. 2003; 20(11):37-45.

26. Luis DA, Bachiller P, Izaola O, Bouza JME, Aller R. Estado nutricional de pacientes infectados por el virus de la inmunodeficiencia humana (VIH). An Med Interna. 2001; 18(12):619-23.

27. Corcoran C, Grinspoon S. Treatments for wasting in patients with the acquired immunodeficiency syndrome. N Eng J Med. 1999; 340(22):1740-50.

28. Highleyman L. Nutrition and HIV. Bolletin of experimental treatments for AIDS. Winter. Foundation San Francisco. AIDS. 2006; 18(2):18-32.

29. Jaime PC, Florindo AA, Latorre MRDO, Brasil BG, Santos ECM, Segurado AAC. Prevalência de sobrepeso e obesidade abdominal em indivíduos portadores de HIVIAIDS, em uso de terapia antiretroviral de alta potência. Rev Bras Epidemiol. 2004; 7(1):65-72.

30. Coppini LZ, Ferrini M T. Síndrome da imunodeficiência adquirida (AIDS). In: Cuppari L, organizador. Guia de nutrição: nutrição clínica no adulto. Barueri: Manole; 2002. p.235-47.

31. Eldridge B. Cuidado nutricional na infecção por HIV e AIDS. In: Mahan LK, Escott-Stump S. Krause, alimentos e dietoterapia. 8a. ed. São Paulo: Roca; 1998. p.848-59.
32. World Health Organization. Reports of a technical consultation. Nutrient requeriments for people living with HIV/AIDS. Geneva: WHO; 2003.

33. Brasil. Ministério da Saúde. Secretaria de Vigilância em Saúde. Programa Nacional de DST/AIDS. Manual clínico de alimentação e nutrição na assistência a adultos infectados pelo HIV. Brasília: Ministério da Saúde; 2006.

34. Polo R. Manual de nutrición y SIDA. 3a. ed. Madrid: Fundación Wellcome España; 2002.

35. Martin JM, González AA, Aguayo PS. Pérdida de peso en el paciente VIH. In: Díaz JP, Emilio LP, Román AR. La infección por el VIH: Guía práctica. 2a. ed. Andalucía: Sociedad Andaluza de Enfermedades Infecciosas; 2000. p.221-32.

36. Food and Nutrition Technical Assistance Project, Academy for Educational Development. HIV/AIDS: a guide for nutritional care and support. 2 nd ed. Washington (DC); 2004. p.10-7.

37. NAM Nutrition. 7th ed. London: NAM, 2006 [cited 2007 Nov 14]. Available from: <http: //www. aidsmap.com/publications/infoseries/nutrition. $\mathrm{pdf}>$.

38. Nicholson M. Diet \& lipodystrophy. In: Positively Aware. Test Positive Aware Network. 2004; 15(3): 23-5.

39. Dreyfuss ML, Fawzi WW. Micronutrients and vertical transmission of HIV-1. Am J Clin Nutr. 2002; 75(6): 959-70.

40. Heaney RP, Rafferty K. Carbonated beverages and urinary calcium excretion. Am J Clin Nutr. 2001; 74(3):343-47.

41. Mondy K, Tebas P. Emerging bone problems in patients infected with human Immunodeficiency virus. Clin Infect Dis. 2003; 36(2):S101-5.

42. Arey BD, Bela MW. The role of exercise in the prevention and treatment of wasting in acquired immunedeficiency syndrome. J Assoc Nurses AIDS Care. 2002; 13(1):29-49.

Recebido em: 27/4/2007

Versão final reapresentada em: 3/4/2008 Aprovado em: 8/4/2008 